\title{
Serial elongation derotation flexion casting in children with infantile and juvenile scoliosis
}

\author{
Federico Canavese $^{1}$, Alain Dimeglio ${ }^{2}$ \\ ${ }^{1}$ Pediatric Surgery Department, University Hospital Estaing, Clermont-Ferrand, France; ${ }^{2}$ Pediatric Orthopedic Department, Clinique St. Roch, \\ Montpellier, France \\ Contributions: (I) Conception and design: All authors; (II) Administrative support: F Canavese; (III) Provision of study materials or patients: All \\ authors; (IV) Collection and assembly of data: All authors; (V) Data analysis and interpretation: All authors; (VI) Manuscript writing: All authors; (VII) \\ Final approval of manuscript: All authors. \\ Correspondence to: Federico Canavese, MD, PhD, Professor of Pediatric Surgery, Pediatric Surgery Department, University Hospital Estaing, 1 Place \\ Lucie et Raymond Aubrac, 63003 Clermont-Ferrand, France. Email: canavese_federico@yahoo.fr.
}

\begin{abstract}
Infantile (IS) and juvenile scoliosis (JS) are among the most challenging conditions pediatric orthopedic surgeons are facing in the present days. However, the best treatment of IS and JS is still debated and it remains controversial, at least for some aspects. Untreated early onset spinal deformities may lead to pulmonary and heart compromise. Growth friendly surgical techniques imply multiple distractions with increased risk of auto-fusion, infection and curve stiffening. Serial casting has been proven to be a valuable option to treat children with early onset scoliosis (EOS) in an attempt to delay surgery (in most cases) or to cure the disease (in few cases). More than five decades ago, Cotrel and Morel from France, introduced the Elongation-Derotation-Flexion (EDF) casting technique. EDF cast is a custom-made thoracolumbar cast that corrects the deformed spine three-dimensionally. Serial EDF casting is able to modulate spinal growth and it can — at least in some cases — prevent the progression of the spinal deformity. Today, serial EDF casting technique has become one of the accepted treatment options for the management of children with IS and JS. The main objective of this work is to describe the EDF serial casting technique for the treatment of children with IS and JS, as well as to highlight its advantages and its limits by providing a review of the most recent literature.
\end{abstract}

Keywords: Infantile (IS) scoliosis; juvenile scoliosis (JS); early onset scoliosis (EOS); Elongation-DerotationFlexion casting (EDF casting); conservative

Submitted Aug 22, 2019. Accepted for publication Aug 27, 2019.

doi: 10.21037/atm.2019.08.108

View this article at: http://dx.doi.org/10.21037/atm.2019.08.108

\section{Introduction}

Scoliosis is a three-dimensional deformity of the spine with lateral, antero-posterior and rotational components. In most cases, the disease is idiopathic and affects children during adolescence. Early onset scoliosis (EOS) affects children younger than 10 years of age. In particular, infantile (IS) and juvenile scoliosis (JS) are diagnosed prior to age 5 and 10 years, respectively and are much rarer compared to adolescent scoliosis (AS). Compared to AS, IS and JS, in addition to age at onset, have higher rate of associated anomalies, as well as frequency and rate of deformity progression (1).

IS and JS are among the most challenging conditions pediatric orthopedic surgeons are facing in the present days. As other forms of EOS, IS and JS carry the potential risk for severe adverse consequences such as cardio-respiratory problems (ventricular tachycardia, chronic obstructive pulmonary disease, tachypnea, dyspnea), tracheomalacia, weight loss or and, in most severe cases, death.

Nowadays, severe forms of IS and JS can be managed with growth-friendly surgical procedures although 


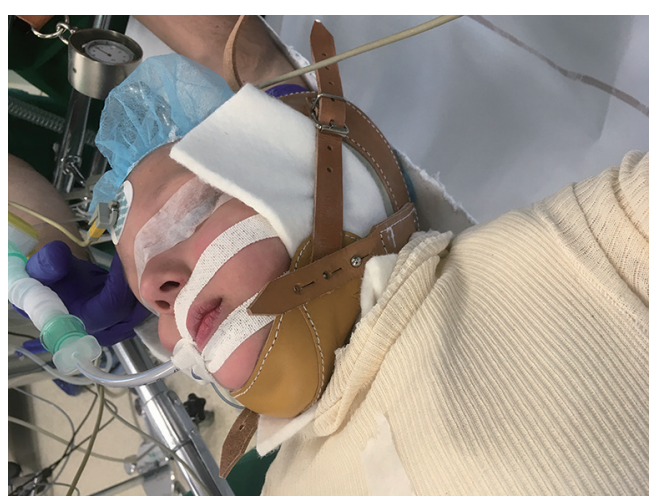

Figure 1 The patient is placed on the Cotrel's frame; the proximal point of traction is represented by the chin and the occiput.

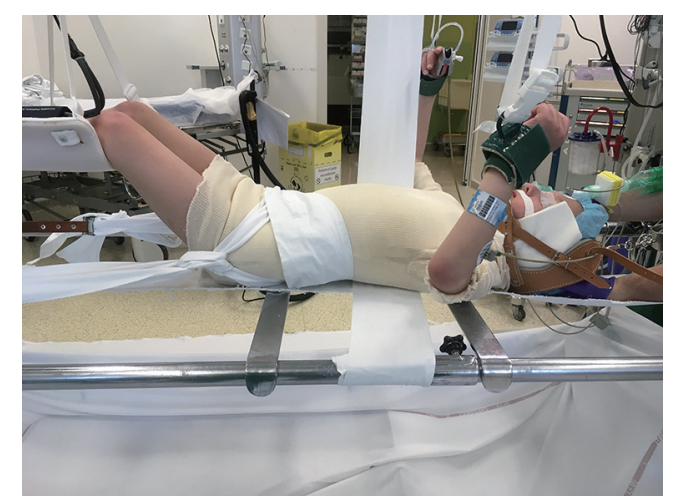

Figure 2 The distal point of traction is represented by the iliac crests, employing of harnesses and bands (lateral view).

multiple distractions are associated with an increased risk of complications such as surgical site infection (superficial and deep), skin problems (dehiscence, keloid), hardware problems (migration, failure), bone-related issues (autofusion, fracture, curve stiffening, decompensation), as well as anesthetic complications. In particular, Mackenzie et al. found that children with IS managed by growing constructs had $29 \%$ surgical site infection rate during lengthening and/or revision surgeries (2). Moreover, repeated hospitalizations for lengthening and for unplanned surgical procedures increase the child's time away from school and can have repercussions on the child's psychological wellbeing $(3,4)$. Moreover, in patients with EOS, early spine fusion carried out in the thoracic spine at an early age does not address the impact of the deformity on thoracic cage shape, lung parenchyma development or preservation of cardio-pulmonary function (5-7). Relative morbidity due to surgical procedures has favored the coming back of conservative treatment, i.e., serial casting.

Although the best treatment option for children with IS and JS has not yet been identified, serial casting has been proven to be a valuable option to treat children with severe spine deformity and thoracic cage distortion secondary to EOS; serial casting can delay and-in some caseseventually avoid surgical treatment.

More than five decades ago (1964), Cotrel and Morel from France, introduced the Elongation-DerotationFlexion (EDF) casting technique by improving the elongation-derotation technique developed by Joseph Risser from United States (8). Few years later (1975), Min Mehta from United Kingdom, in order to accommodate the growth of the child, developed the concept of serial casting (cast changes every $8-12$ weeks under general anesthesia) $(8,9)$.

EDF cast is a custom-made thoracolumbar cast that corrects the deformed spine three-dimensionally. Serial EDF casting is able to modulate spinal growth and it canat least in some cases-prevent the progression of the spinal deformity. Spinal growth can be guided by EDF casting ("positive force" to guide spinal growth) as it can influence the initially curved spine to grow straighter $(8,9)$.

The main objective of this work is to describe the EDF serial casting technique for the treatment of children with IS and JS, as well as to highlight its advantages and its limits by providing a review of the most recent literature.

\section{Serial elongation, derotation, and flexion casting technique}

The EDF technique is a non-surgical method to reduce the spinal deformity three-dimensionally. It requires a special frame (the so called Cotrel's frame) in order to apply at the same time (I) traction (Elongation); (II) postero-lateral compression and rotation (Derotation); and (III) manual lateral compression (Flexion) (8).

The patient is supported by two horizontal metal bars, one under the shoulders and the other sustaining the pelvis. The axial correction (Elongation) of the spine is obtained with traction. The proximal traction is fixed at the chin and at the occiput while the distal traction is fixed at both iliac crests (8) (Figure 1). Standard stockinette and traditional padding are used to protect the skin of the patient (Figure 2). Simultaneous posterolateral compression and rotation (Derotation) are obtained with a band surrounding the apex of the spinal curvature (convex side); in the case of a double curve, a second band can be applied in the opposite direction and side, on the level of 


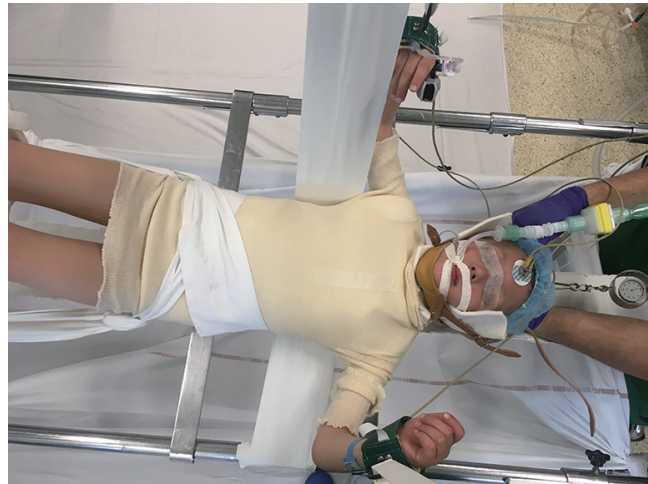

Figure 3 The patient is supported by two metal bars (top view), one under the shoulders and the other sustaining the pelvis. A band surrounds the apex of the spinal curvature (convex side); in the case of a double curve, a second band can be applied in the opposite direction and side, on the level of the convexity of the second curve.

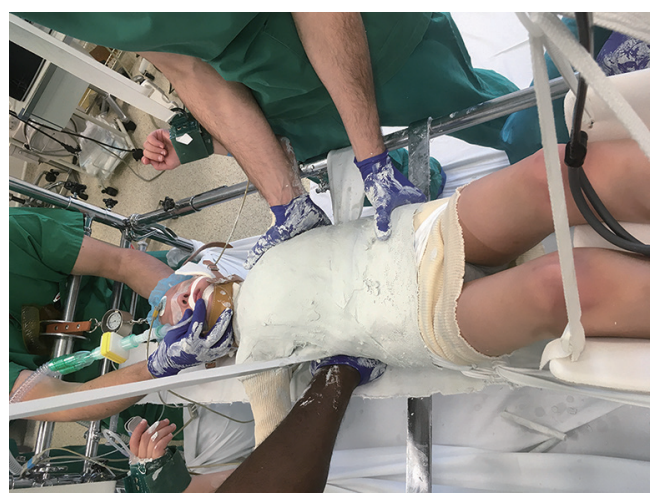

Figure 4 Manual lateral compression is applied while the plaster is still malleable. One hand (first surgeon) is required to push against the apex of the deformity (convex side) while two hands (second surgeon) apply a counter-pressure as closed as possible to the upper and lower end vertebra of the scoliotic deformity (concave side); pressure is maintained until the plaster hardens.

the convexity of the second curve (Figure 3).

At this point the two support bars are removed and several layers of plaster of Paris (POP) are applied over the bands. The POP needs to be well molded, especially at the level of both iliac crests: a solid and stable foundation of the cast in needed to obtain a comfortable cast and to avoid pressure sores. Manual lateral compression (Flexion) is applied while the plaster is still malleable. At this point, one hand (first surgeon) is required to push against the apex of the deformity (convex side) while two hands (second surgeon) apply a counter-pressure as closed as possible to the upper and lower end vertebra of the scoliotic deformity (concave side); pressure is maintained until the plaster hardens (Figure 4). At the same time over the shoulder straps are applied (Figure 5).

Once the POP has hardened, it can be reinforced by a two to three layers of synthetic fiberglass (Figure 6); at this stage a thoraco-abdominal window is cut as to obtain a decompression of the anterior abdomen (stomach and bowels) and a better expansion of the thoracic cage (Figure 7).

The casts are changed at intervals of 8 to 12 weeks in order to accommodate the growth of the child. On average, it takes between 20 to 35 minutes to realize an EDF cast on the Cotrel's frame, depending on the height and weight of the patient.

Some surgeons suggest the use of POP only due to his better molding ability compared to fiberglass, despite the fact that the resulting cast is generally heavier and more prone to breakage (10-12). On the other hand, in some other Institutions casts are made of fiberglass only (light and durable material) although Daines et al. reported that it is stiffer and more difficult to mold compared to POP (11). The use of a hybrid POP (deep layers) and fiberglass (superficial layers) may have the advantage of decreasing the overall body cast weight and to enhance its resistance (13).

Fedorak et al. compared the outcome of two groups of children with EOS: Group 1 included patients $(n=40)$ that underwent radiographs after each cast and had the cast reinforced with over the shoulder straps while Group 2 included children $(n=36)$ that had radiographs taken every 6 months and cast was not reinforced with over the shoulder straps. They found that children in Group 1 ( $\mathrm{n}=9$ resolution of scoliosis; $22.5 \%$ ) had results very similar to the children of Group 2 ( $\mathrm{n}=11$ resolution of scoliosis; $30.5 \%$ ) and concluded the over the shoulder straps and frequent radiographs are not needed (14). However, the original EDF cast technique requires the application of over the shoulder straps (8).

At first sight, cast may appear as a constrictive force applied to the thoracic cage, limiting its expansion. Indeed, if well molded it does not compress the thoracic cage and respiratory movements are allowed. In particular, Dhawale et al. investigated the effects of casting on ventilation in IS. They reviewed data from 37 serial EDF casts. They found that casting induced transient restrictive pulmonary process: peak inspiratory pressure increased by $106 \%$ at cast application and remained $32 \%$ higher than baseline after thoraco-abdominal winds were cut off (15). 


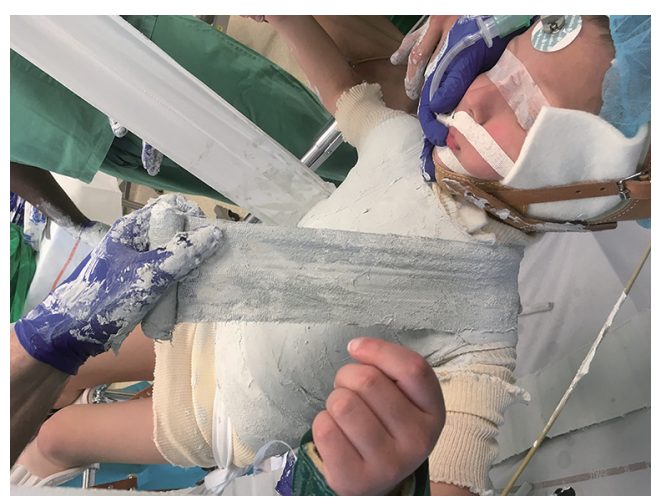

Figure 5 Over the shoulder straps are applied prior the EDF cast complete hardening. EDF, Elongation-Derotation-Flexion.

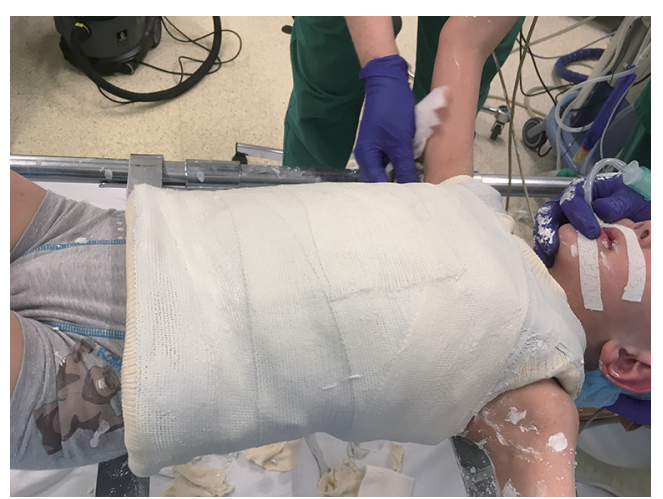

Figure 6 The plaster of Paris cast is reinforced with several layers of fiberglass (two to three).

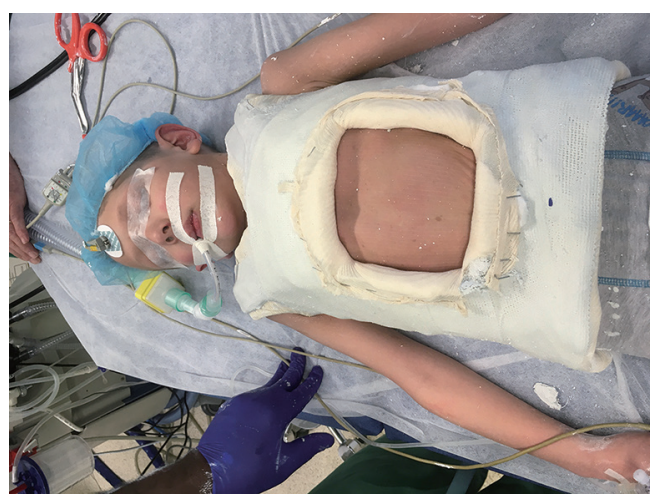

Figure 7 An anterior opening is realized.

\section{Serial EDF casting: delaying tactic or definitive treatment option?}

The impact of a progressive, severe EOS on a growing organism can be devastating. A vertebral column not permitted to grow normally, will affect the growth potential of the whole upper body, resulting in a short trunk, a disproportionate body habitus and an underdeveloped thoracic cage (the so called 'domino effect' as described by Canavese et al.) (7,16-18). In addition, Pehrsson et al. investigated the mortality rate in patients $(\mathrm{n}=115)$ with untreated scoliosis and compared it to the expected mortality rate of official Swedish statistics. They found that the mortality rate was significantly increased in patients with IS and JS but not in those with AS (19).

The serial EDF casting technique can be used either as delaying tactic, in order to prevent deformity progression, or as definitive treatment option. It has been shown that patients with IS casted prior to age 20 months had their scoliosis non progressing and/or significantly reduced at the end of skeletal growth (from $30^{\circ}$ to $10^{\circ}$, on average). On the other hand, children with IS averaging $50^{\circ}$ that were treated after age 30 months had their spinal curvature not progressing, although no significant and stable correction could be achieved (9). Serial casting is a non-surgical alternative growth sparing techniques that can potentially help to delay surgery in children with moderate-to-severe IS and JS (19).

Waldron et al. reviewed 20 consecutive patients with EOS treated by serial casting (Risser's technique). Among the 20 included patients, 8 had idiopathic IS (40\%) and 12 had non-idiopathic EOS (60; $\mathrm{n}=6$ neuromuscular scoliosis, $\mathrm{n}=5$ syndromic scoliosis, $\mathrm{n}=1$ skeletal dysplasia). The mean age at first cast was 3.8 years $(1-8)$, and the mean major curve magnitude was $74^{\circ}\left(40^{\circ}-118^{\circ}\right)$. During treatment 4 patients experienced minor complications $(20 \%)$ such as superficial skin irritation ( 2 cases) and cast intolerance (2 cases). Waldron et al. concluded that serial casting is a safe delaying technique as it can both stabilize the spinal deformity and allow the child to reach a more suitable age for other forms of treatment, such as growing rods (20).

The main advantage of EDF casting is that the spine is left alone. Implantation of growth sparing devices, even if at reasonable distance from the spine, does not decrease 
the surgical risk, does not protect from auto-fusion (ribs, vertebrae) and does not facilitate definitive instrumented fusion $(3,6,16,18,21)$.

Opposite to surgery, serial EDF casting is an alternative which does not negatively affect spinal growth. Serial EDF casting is able to modulate spinal growth and it can-at least in some cases - prevent the progression of the spinal deformity. Spinal growth can be guided by EDF casting ("positive force" to guide spinal growth) as it can influence the initially curved spine to grow straighter $(8,9,22)$. However, serial EDF casting is not effective for all types of IS and JS.

Between early 50's and late 60's few works have been published in the English literature. Scott and Morgan reported that resolving curves are not uncommon (23) while Conner demonstrated that EOS associated with developmental anomalies are more prone to progress (24). However, James et al. (25) and Lloyd-Roberts and Pilcher (26) could not identify absolute criteria for distinguishing between resolving and progressive curves.

In 1972, Mehta showed patients with IS casted prior to age 20 months had their scoliosis non progressing and/ or significantly reduced at the end of skeletal growth (from $30^{\circ}$ to $10^{\circ}$, on average). On the other hand, children with IS averaging $50^{\circ}$ that were treated after age 30 months had their spinal curvature not progressing, although no significant and stable correction could be achieved $(9,27)$. Ceballos et al. reviewed 113 patients with IS and found, as previously reported by Metha, the relationship of the rib head and its corresponding vertebral body at the apex of the spinal deformity is the most reliable prognostic radiologic factor $(27,28)$.

In a more recent article, Mehta retrospectively reviewed 136 children with IS. She found heterogeneous results although all patients were managed with the same protocol. In particular in children treated before age 2 years the scoliosis resolved $(94 / 136 ; 69.1 \%)$. On the other hand, patients treated after the age of 2.5 years had the deformity reduced but not cured $(42 / 136 ; 30.9 \%)$; the rate of surgery in this subgroup of patients was $37.5 \%$. Mehta pointed out that avoidance of delay in treatment is of crucial importance (9).

Sanders et al. reviewed 55 patients with progressive IS scoliosis treated by means of derotational casting. They found that $84 \%$ of patients $(46 / 55)$ responded to treatment and, at the time of publication, did not require surgical intervention. Sanders et al. confirmed Mehta's findings $(9,27,29)$ that patients with moderate idiopathic scoliosis treated at younger age, have a better prognosis compared to children with more sever $\left(>60^{\circ}\right)$ and/or non-idiopathic curves (29).

Fletcher et al. and Baulesh et al. have retrospectively reviewed a group of 29 and 36 patients with IS treated with derotational casting, respectively. Although a definitive cure could not be obtained in this cohort of patients, Fletcher et $a l$. and Baulesh et al. reported an average 39 and 25 months of delay of surgery, respectively. Moreover, about two thirds of patients in both series $(72 \%$ and $69 \%$, respectively) did not require surgical intervention. Fletcher et al. and Baulesh et al. both concluded that serial casting is a valid alternative to surgical growth sparing techniques in moderate-to-severe EOS and may help delay eventual surgery (30,31). In addition, Baulesh et al. showed that serial casting is able to preserve normal longitudinal thoracic growth in patients with EOS. Although about one third of patients required surgery, they concluded that the increased thoracic height may have positive implications on ultimate pulmonary function (31). Similarly, Morin and Kulkarni reported that serial EDF casting for the treatment of progressive idiopathic IS is an effective treatment method for the Metha's benign-type of curves $(9,24)$ and spinal fusion can be potentially avoided in about $70 \%$ of cases (32).

Johnston et al. reviewed 27 patients with IS and JS treated with serial casting. Although no distinction was made between IS and JS, and idiopathic patients were not analyzed separately from patients with syndromic diagnoses, the mean Cobb angle remained stable after a mean duration of treatment of 28 months. Despite study limitations, Johnston et al. concluded that cast treatment is a valuable delaying tactic for children with EOS as it does not compromise spinal length, it can adequately control spine deformity, and it avoids surgical complications associated with growth sparing surgery (33).

Welborn et al. investigated which factors predict successful outcomes in children with IS treated by serial EDF casting. They analyzed 31 children with idiopathic IF and none of them progressed to surgical intervention during the study period. They found that major Cobb angle at the end of casting is most predictive of outcome whereas magnitude of initial curvature, earlier age at onset of casting, flexibility, initial correction, sex, and curve type were not found to predict successful outcomes. Welborn et al. concluded that serial EDF casting is a valid treatment option for IS with a high cure rate (34).

Similarly, Hassanzadeh et al. retrospectively evaluated radiographs of 45 children with IF treated by serial casting (Metha) and attempted to identify the radiographic factors potentially correlated with a sustained curve correction. In particular, they evaluated Cobb angle, focal deformity, rib-vertebral angle difference, and height of concavity and 
convexity of the apical three vertebrae. They found that the most significant radiographic parameters associated with control of progressive deformity include improvements in focal deformity and height of concavity and convexity of the apical three vertebrae (35).

On the other hand, casting and especially bracing has been less successful in children with JS compared to those with IS, with reported surgery rates ranging from $27 \%$ to $100 \%$ after conservative treatment. In particular, most studies have highlighted that most loss of correction tend to occur between fitting point and end of treatment (with curve magnitude similar to initial treatment) and after the weaning point (further aggravation of curve magnitude) (36-43).

More recently, van Hessem $e t$ al. retrospectively reviewed 7 patients with JS treated by serial casting and reported Cobb's angle was reduced from $37^{\circ}$ to $25^{\circ}$ (32\% reduction) and none of the patients required surgery at a mean followup of 4.6 years. They concluded that serial casting can stop curve progression and it can reduce the need for surgery in patients with JS (22).

\section{Is there a role for neuromuscular-blocking drugs (NBD) during EDF cast application?}

Recently, Canavese et al. reviewed a cohort of 44 patients with JS. They reported that serial EDF casting under general anesthesia with NBD, i.e., curare, is more effective in controlling curve progression in patients with JS, compared to EDF casting under general anesthesia alone or no anesthesia.

Canavese et al. hypothesized that complete muscle relaxation may allow the surgeon to obtain a better threedimensional correction of the spinal deformity as curve magnitude, vertebral rotation and rib vertebral angle difference (27) particularly improved in patients with serial EDF casting performed under general anesthesia with NBD $(44,45)$.

Canavese et al. concluded that serial EDF casting and general anesthesia with NBD is a safe technique. It provides better initial correction compared to serial EDF casting with patients awake and to serial EDF casting with general anesthesia alone (44).

\section{Does serial casting work for congenital EOS?}

The therapeutic efficacy of serial casting on congenital scoliosis is scarce.

Demirkiran et al. reviewed 11 patients with progressive congenital scoliosis treated by serial casting (age at initial treatment: 40 months; average number of cast changes: 6.2). At the time of last follow-up the main curve (coronal plane) decreased from $70^{\circ}$ to $55^{\circ}$ on average, while the compensatory curves (coronal plane) decreased from $56^{\circ}$ to $40^{\circ}$, on average; improvement was also observed on the sagittal plane (from $76^{\circ}$ to $64^{\circ}$ on average). Interestingly, during treatment period none of the patients required surgery for curve progression nor developed complications. Demirkiran et al. concluded that serial derotational casting is a safe and effective strategy to delay surgery (26 months on average) in children with congenital deformities. However, the potential benefits of serial casting in children with congenital scoliosis have been shown in the short-term follow-up only (46).

More recently, Cao et al. compared the treatment outcomes of serial casting between congenital scoliosis $(n=8)$ and non-congenital scoliosis patients $(n=15)$. They found that although Cobb angle improved in both groups, the correction rate was significantly higher in non-congenital scoliosis (50\%) group than in congenital scoliosis group (20\%). Despite the fact that therapeutic efficacy of casting on congenital scoliosis patients is not as good as that on non-congenital scoliosis patients, serial casting remains a valuable option for delaying surgery (47).

\section{Complications of serial casting}

Serial casting has a very low rate of complications. The most common complication of serial casting are minor skin lesions (superficial pressure sores) that do not require treatment in most cases. Ventilatory problems have been described following cast application however they soon resolved after cast removal (rare). Increased peak inspiratory pressures during the cast application have been reported by Dhawale et al. In particular they found that following cast application, the mean peak inspiratory pressure increased by $106 \%$ when compared with the baseline value and remained 32\% higher than baseline following the removal of the thoracoabdominal winds (15).

Careful trimming of the cast in the axillary and groin regions is necessary in order to avoid subclavian vein thrombosis, an extremely rare but serious complication of serial casting (48).

\section{Conclusions}

Serial EDF casting should be considered a valuable low risk 
treatment modality, as well as an alternative to surgery, for patients with IS and JS.

Serial EDF casting is able to modulate spinal growth and it can-at least in some cases-prevent the progression of the spinal deformity. However, it controls all types of curves and the technique is not able to completely arrest curve progression and to erase the potential need for surgery in this patient's population.

Despite these limitations, it is effective in delaying a sometimes eliminating the need for growth-sparing surgery. Unfortunately, there is a lack of scientific publications reporting long term outcome of serial EDF casting.

EDF casting is a safe technique that can modify the natural evolution of IS and JS by reducing and slowing curve progression in both frontal and transverse plane.

\section{Acknowledgments}

None.

\section{Footnote}

Conflicts of Interest: The authors have no conflicts of interest to declare.

Ethical Statement: The authors are accountable for all aspects of the work in ensuring that questions related to the accuracy or integrity of any part of the work are appropriately investigated and resolved.

\section{References}

1. Koop SE. Infantile and juvenile idiopathic scoliosis. Orthop Clin North Am 1988;19:331-7.

2. Mackenzie WG, Matsumoto H, Williams BA, et al. Surgical site infection following spinal instrumentation for scoliosis. J Bone Joint Surg Am 2013;95:800-6.

3. Lattig F, Taurman L, Hell AK. Treatment of Early Onset Spinal Deformity (EOSD) with VEPTR: A Challenge for the Final Correction Spondylodesis: A Case Series. Clin Spine Surg 2016;29:E246-51.

4. Bess S, Akbarnia BA, Thompson GH et al. Complications of growing rod treatment for early-onset scoliosis: analysis of one hundred and forty patients. J Bone Joint Surg Am 2010;92:2533-43.

5. Canavese F, Dimeglio A, Volpatti D, et al. Dorsal arthrodesis of thoracic spine and effects on thorax growth in prepubertal New Zealand white rabbits. Spine
2007;32:E443-50.

6. Karol LA, Johnston CE, Mladenov K, et al. Pulmonary function following early thoracic fusion in nonneuromuscular scoliosis. J Bone Joint Surg Am 2008;90:1272-81.

7. Dimeglio A, Canavese F. The growing spine: how spinal deformities influence normal spine and thoracic cage growth. Eur Spine J 2012;21:64-70.

8. Cotrel Y, Morel G. The elongation-derotation-flexion technique in the correction of scoliosis. Rev Chir Orthop Reparatrice Appar Mot 1964;50:59-75.

9. Mehta MH. Growth as a corrective force in early treatment of progressive infantile scoliosis. J Bone Joint Surg Br 2005;87:1237-47.

10. DeMaio M, McHale K, Lenhart M, et al. Plaster: our orthopedic heritage: AAOS exhibit selection. J Bone Joint Surg Am 2012;94:e152.

11. Daines SB, Aronsson DD, Beynnon BD, et al. What is the best material for molding casts in children? J Pediatr Orthop 2014;34:743-8.

12. Kowalski KL, Pitcher JD Jr, Bickley B. Evaluation of fiberglass versus plaster of Paris for immobilization of fractures of the arm and leg. Mil Med 2002;16:657-61.

13. Charles MN, Yen D. Properties of a hybrid plasterfiberglass cast. Can J Surg 2000;43:365-7.

14. Fedorak GT, Stasikelis PJ, Carpenter AM, et al. Optimization of Casting in Early-onset Scoliosis. J Pediatr Orthop 2019;39:e303-7.

15. Dhawale AA, Shah S, Reichard S, et al. Casting for infantile scoliosis: the pitfall of increased peak inspiratory pressure. J Pediatr Orthop 2013;33:63-7.

16. Canavese F, Dimeglio A, Stebel M, et al. Thoracic Cage Plasticity in prepubertal New Zealand White Rabbits Submitted to T1-T12 Dorsal Arthrodesis: Computed Tomography Evaluation, Echocardiographic Assessment and Cardio-pulmonary Measurements. Eur Spine J 2013;22:1101-12.

17. Dimeglio A. Growth of the spine before age 5 years. J Pediatr Orthop B 1992;1:102-7.

18. Canavese F, Dimeglio A, Bonnel F, et al. Thoracic cage volume and dimension assessment by optoelectronic molding in normal children and adolescents during growth. Surg Radiol Anat 2019;41:287-96.

19. Pehrsson K, Larsson S, Oden A, et al. Long-term followup of patients with untreated scoliosis. A study of mortality causes of death, and symptoms. Spine 1992;17:1091-6.

20. Waldron SR, Poe-Kochert C, Son-Hing JP, et al. Early onset scoliosis: the value of serial risser casts. J Pediatr 
Orthop 201;33:775-80.

21. Zivkovic V, Buchler P, Ovadia D, et al. Extraspinal ossifications after implantation of vertical expandable prosthetic titanium ribs (VEPTRs). J Child Orthop 2014;8:237-44.

22. van Hessem L, Schimmel JJ, Graat HC, et al. Effective nonoperative treatment in juvenile idiopathic scoliosis. J Pediatr Orthop B 2014;23:454-60.

23. Scott JL, Morgan TH. The natural history and prognosis of infantile idiopathic scoliosis. J Bone Joint Surg Br 1955;37-B:400-13.

24. Conner AN. Developmental anomalies and prognosis in infantile idiopathic scoliosis. J Bone Joint Surg Br 1969;51:711-3.

25. James JI, Lloyd-Roberts GC, Pilcher MF. Infantile structural scoliosis. J Bone Joint Surg Br 1959;41-B:719-35.

26. Lloyd-Roberts GC, Pilcher MF. Structural idiopathic scoliosis in infancy: a study of the natural history of 100 patients. J Bone Joint Surg Br 1965;47:520-3.

27. Mehta MH. The rib-vertebral angle in the early diagnosis between resolving and progressive infantile scoliosis. J Bone Joint Surg Br 1972;54:230-43.

28. Ceballos T, Ferrer-Torrelles M, Castillo F, et al. Prognosis in infantile idiopathic scoliosis. J Bone Joint Surg Am 1980;62:863-75

29. Sanders JO, D’Astous J, Fitgerald M, et al. Derotational casting for progressive infantile scoliosis. J Pediatr Orthop 2009;29:581-7.

30. Fletcher ND, McClung A, Rathjen KE, et al. Serial casting as a delaying tactic in the treatment of moderate-to-severe early onset scoliosis. J Pediatr Orthop 2012;32:664-71.

31. Baulesh DM, Huh J, Judkins T, et al. The role of serial casting in early-onset scoliosis (EOS). J Pediatr Orthop 2012;32:658-63.

32. Morin C, Kulkarni S. ED plaster-of-Paris jacket for infantile scoliosis. Eur Spine J 2014;23:S412-8.

33. Johnston CE, McClung AM, Thompson GH, et al. Comparison of growing rod instrumentation versus serial cast treatment of early-onset scoliosis. Spine Deform 2013;1:339-42.

34. Welborn MC, D'Astous J, Bratton S, et al. Infantile Idiopathic Scoliosis: Factors Affecting EDF Casting Success. Spine Deform 2018;6:614-20.

35. Hassanzadeh H, Nandyala SV, Puvanesarajah V, et al. Serial Mehta Cast Utilization in Infantile Idiopathic Scoliosis: Evaluation of Radiographic Predictors. J Pediatr Orthop 2017;37:387-91.

36. Grivas TB, Kaspiris A. European braces widely used for conservative scoliosis treatment. Stud Health Technol Inform 2010;158:157-66.
37. Lonstein JE, Winter RB. The Milwaukee brace for the treatment of adolescent idiopathic scoliosis. A review of one thousand and twenty patients. J Bone Joint Surg Am 1994;76:1207-21.

38. Gabos PG, Bojescul JA, Bowen JR, et al. Long-term follow-up of female patients with idiopathic scoliosis treated with the Wilmington orthosis. J Bone Joint Surg Am 2004;86:1891-9.

39. Dabney KW, Browen JR. Juvenile idiopathic scoliosis. Semin Spine Surg 1991;3:524-30.

40. Tolo VT, Gillespie R. The characteristics of juvenile idiopathic scoliosis and results of treatment. J Bone Joint Surg Br 1978; 60-B:181-8.

41. Coillard C, Leroux MA, Zabjek KF, et al. SpineCor nonrigid brace for the treatment of idiopathic scoliosis: posttreatment results. Eur Spine J 2003;12:141-8.

42. Robinson CM, McMaster MJ. Juvenile idiopathic scoliosis. Curve patterns and prognosis in one hundred and nine patients. J Bone Joint Surg Am 1996;78:1140-8.

43. Kahanovitz N, Levine DB, Lardone J. The parttime Milwaukee brace treatment of juvenile idiopathic scoliosis. Long-term follow-up. Clin Orthop Relat Res 1982;167:145-51.

44. Canavese F, Botnari A, Dimeglio A, et al. Serial elongation, derotation and flexion (EDF) casting under general anesthesia and neuromuscular blocking drugs improve outcome in patients with juvenile scoliosis: preliminary results. Eur Spine J 2016;25:487-94.

45. Canavese F, Holveck J, De Coulon G, et al. Analysis of concave and convex rib-vertebral angle, angle difference and angle ratio in patients with Lenke type I main thoracic adolescent idiopathic scoliosis treated by observation, bracing or posterior fusion and instrumentation. J Spinal Disord Tech 2011;24:506-13.

46. Demirkiran HG, Bekmez S, Celilov R, et al. Serial derotational casting in congenital scoliosis as a time buying strategy. J Pediatr Orthop 2015;35:43-9.

47. Cao J, Zhang XJ, Sun N, et al. The therapeutic characteristics of serial casting on congenital scoliosis: a comparison with non-congenital cases from a single-center experience. J Orthop Surg Res 2017;12:56.

48. Badlani N, Korenblit A, Hammerberg K. Subclavian vein thrombosis after application of body cast. J Pediatr Orthop 2013;33:e1-3.

Cite this article as: Canavese F, Dimeglio A. Serial elongation derotation flexion casting in children with infantile and juvenile scoliosis. Ann Transl Med 2020;8(2):24. doi: 10.21037/ atm.2019.08.108 